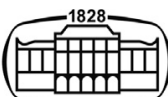

AKADÉMIAI KIADÓ

IMAGING

\section{CASE REPORT}

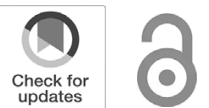

IMAGING 13 (2021) 1, 76-79

DOI: $10.1556 / 1647.2021 .00004$

(c) 2021 The Author(s)

* Corresponding author. Department of Neuroradiology, Faculty of Medicine Medical Imaging Centre, Semmelweis University, Korányi Sándor Street 2, 1083, Budapest, Hungary. Tel.: +36 20/666 3274.

E-mail: magyar.mate@med.semmelwe is-univ.hu

\title{
Liver displacement caused by distended stomach in Parkinsonian patient
}

\author{
MÁTÉ MAGYAR ${ }^{1 *}$ (D), TIBOR GLASZ ${ }^{2}$, TEKLA KOVÁCS ${ }^{2}$, \\ ANDRÁS BÁLINT DEÁK ${ }^{2}$, PÁL MAUROVICH-HORVAT ${ }^{3}$ and \\ BALÁZS FUTÁCSI ${ }^{3}$
}

\author{
${ }^{1}$ Department of Neuroradiology, Faculty of Medicine, Medical Imaging Centre, Semmelweis \\ University, Budapest, Hungary \\ ${ }^{2}$ 2nd Department of Pathology, Faculty of Medicine, Semmelweis University, Budapest, Hungary \\ ${ }^{3}$ Department of Radiology, Faculty of Medicine, Medical Imaging Centre, Semmelweis University, \\ Budapest, Hungary
}

Received: June 25, 2020 • Accepted: December 16, 2020

\section{ABSTRACT}

Gastroparesis, a form of gastrointestinal dysfunction and the increased risk for aspiration pneumonia are well known complications in patients with Parkinson's disease. In our case report we demonstrate the case of an 83-year-old, bedridden male patient with Parkinson's disease, who had a slowly progressing stomach dilation, gradually pushing the otherwise normal liver to a highly unusual craniodorsally rotated position.

\section{KEYWORDS}

Parkinson's disease, stomach, gastroparesis, pneumonia

\section{Case}

The 83-year-old bedridden male patient treated with Parkinson's disease and low-stage prostate cancer was admitted to the Emergency Department with diffuse abdominal pain and hematemesis for four days. Tachypnea, pain, sluggish bowel sounds and blood-stained black vomitus were found at physical examination.

Lab results showed augmented white blood cell counts with mildly elevated CRP levels. Abdominal ultrasonography found the diaphragm in an extremely high position, without fluid accumulation in the abdominal cavity.

Chest X-ray ruled out perforation, but also revealed an enormous, gas filled, hollow organ in the epigastrium causing the extreme elevation of the right hemi-diaphragm (Fig. 1). Consequently, our team performed an abdominal CT- examination and identified the gas filled organ as the atonic, enlarged stomach that pushed and rotated the liver cranially and dorsally by $90^{\circ}$ (Fig. 2). To clear this rotation and dislocation of the liver, we present two comparable CT images in sagittal plane with normal anatomical conditions, and with the abnormal, rotated, and dislocated liver position (Fig. 3).

To relive the distension, a nasogastric tube was inserted in the Emergency Department ward and a large amount of black detritus was drained off. However, right after the procedure, the patient became restless, hypotonic and hypoxic. Endotracheal intubation and assisted respiration were immediately performed. Despite these efforts, ECG showed cardiac arrest and despite advanced lifesaving measures the patient's life could not be saved.

Postmortem examination confirmed the diagnosis of an exceptionally distended stomach (Fig. 4) and an otherwise normal, but cranially and dorsally displaced and rotated liver. 


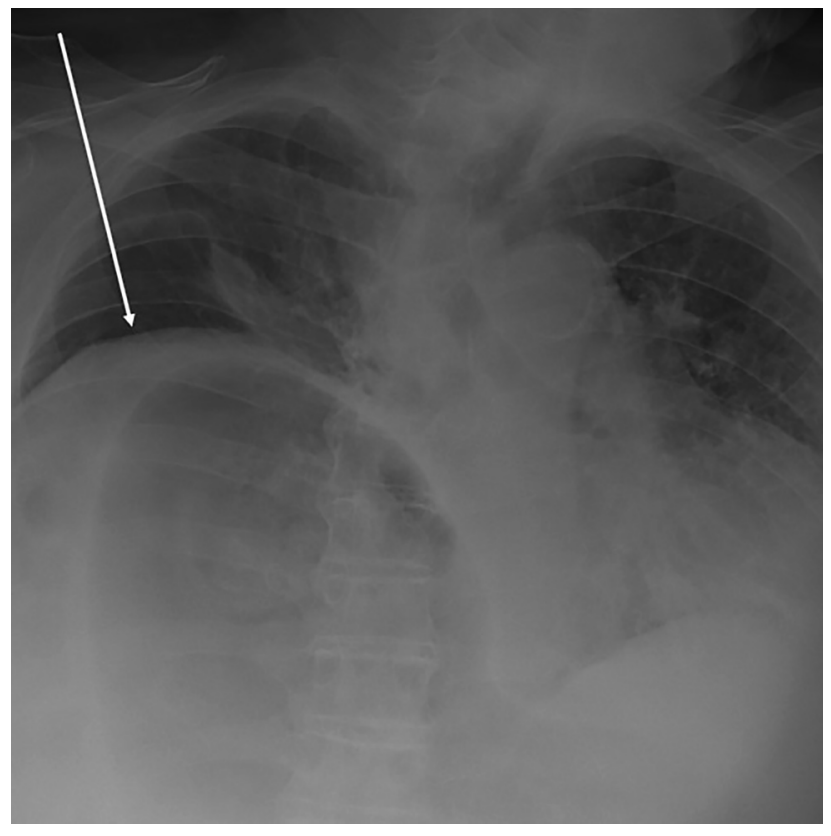

Fig. 1. Patient's last chest X-ray showing an enormous, gas filled stomach and elevated right hemi-diaphragm

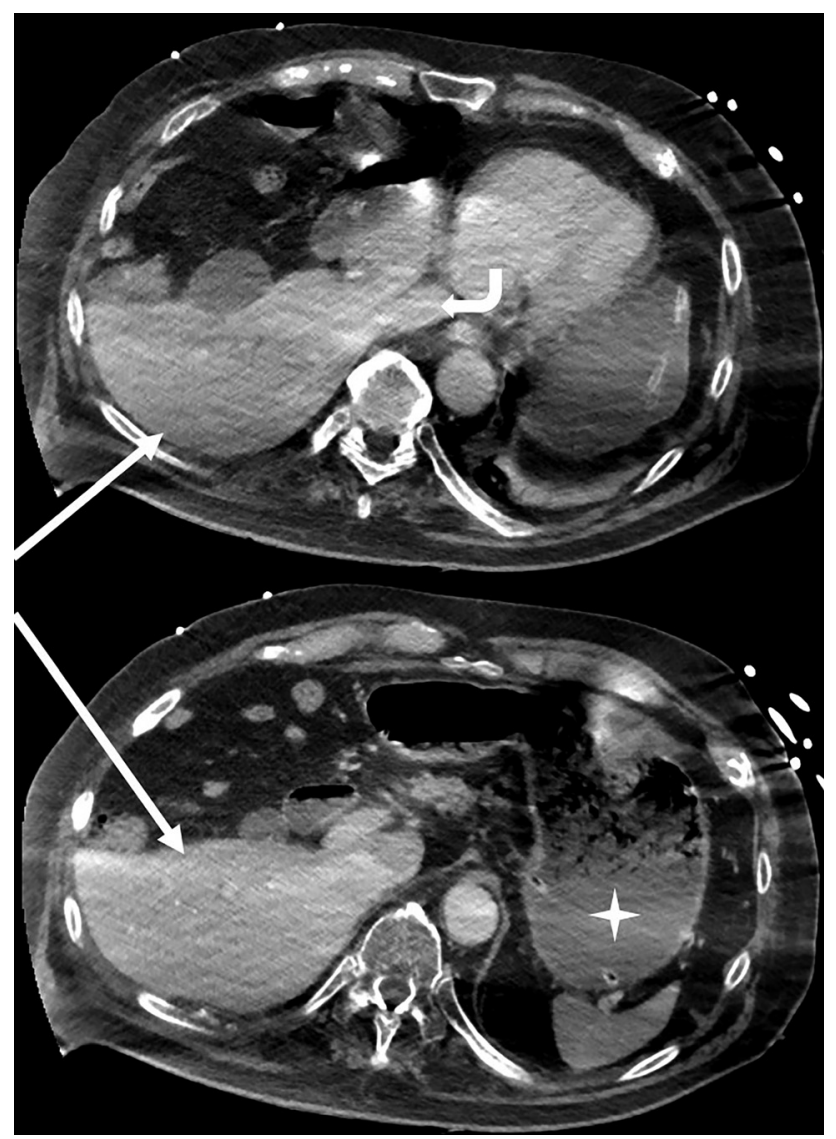

Fig. 2. Abdominal contrast-enhanced CT in axial planes identified gas filled, atonic stomach (asterisk), misplaced and rotated liver (single arrow), and elongated inferior vena cava (curved arrow). No liver can be seen, at the ventral part of the abdomen
The pathological assessment suggested that aspiration of the gastric retention, consecutive gangrenous pneumonia and the compression of the right lower lung lobe (caused by the diaphragmatic elevation due to the stomach dilation and the displaced liver) resulted in a sudden respiratory failure, the direct cause of death.

\section{Discussion}

Patients with Parkinson's disease have an increased risk of developing aspiration pneumonia [1-3] resulting from decreased cough sensitivity [4] and sialorrhea, causing continuous saliva aspiration [5]. Gastrointestinal dysfunctions, including gastroparesis are well known in advancedstage Parkinson's disease [6-8].

Our patient never had abdominal surgery. Previously, he had chest X-rays in 2008 and 2014, the latter - performed 5 years (!) before his death - already showing a highly elevated right hemi-diaphragm, leveling at the pulmonary hilum (Fig. 5). Abdominal ultrasound examinations - also performed in 2008 and 2014 - found a normal and homogeneous liver, only visible from the intercostal space.

In the literature, causes for liver displacement are diaphragmatic herniation [9] and dislocation caused by the large intestines or the stomach [10]. In these, and probably similar cases functional impairment or intermittent jaundice may or may not occur and patients are usually symptom free.

Our hypothesis is that the patient had a slowly progressing stomach dilation that pushed the liver into its highly unusual, $90^{\circ}$ craniodorsally rotated position. While being gradual, it has not compromised its circulation or bile excretion. Besides mechanical displacement, developmental malposition and malrotation can also be considered. One clue for the developmental theory could be that even though gastroparesis is well documented in advanced-stage Parkinson's disease, we could not find any similar cases in the literature; where a largely distended stomach and a highly unusual malposition of the liver were described as above. Whatever the cause may be, it should be noted that this abnormal anatomical position of the liver could be a decisive, indirect factor in the respiratory dysfunction, leading to the patient's death.

Authors' contribution: All persons who meet authorship criteria are listed as authors, and all authors certify that they have participated sufficiently in the work to take public responsibility for the content, including participation in the concept, writing, or revision of the manuscript. Acquisition of images: MM, TK, TK, ABD. Interpretation of literature: MM. Drafting the manuscript: MM, TK, TK, ABD. Revising the manuscript critically for important intellectual content: TK, TK, ABD, BF, PMH. Approval of the version of the manuscript to be published: $\mathrm{PMH}, \mathrm{BF}, \mathrm{TK}, \mathrm{TK}, \mathrm{ABD}, \mathrm{MM}$. This manuscript has not been published previously and is not under consideration for publication elsewhere. 


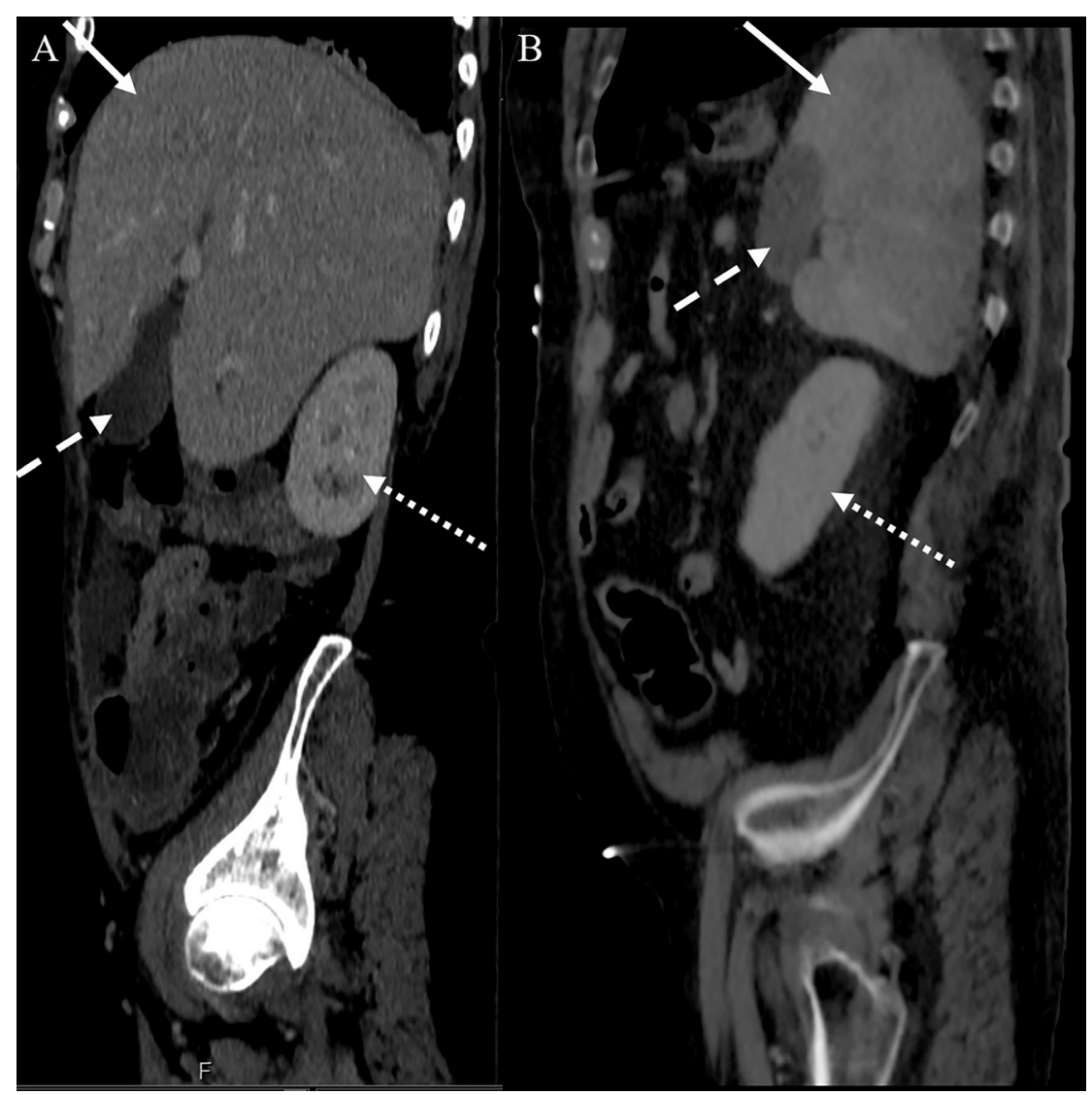

Fig. 3. Comparable sagittal plane CT reconstructions with normal anatomy (A) and with the rotated, dislocated liver position (B). Arrow with continuous line: liver. Arrow with dotted line: gallbladder. Arrow with dotted stem: right kidney

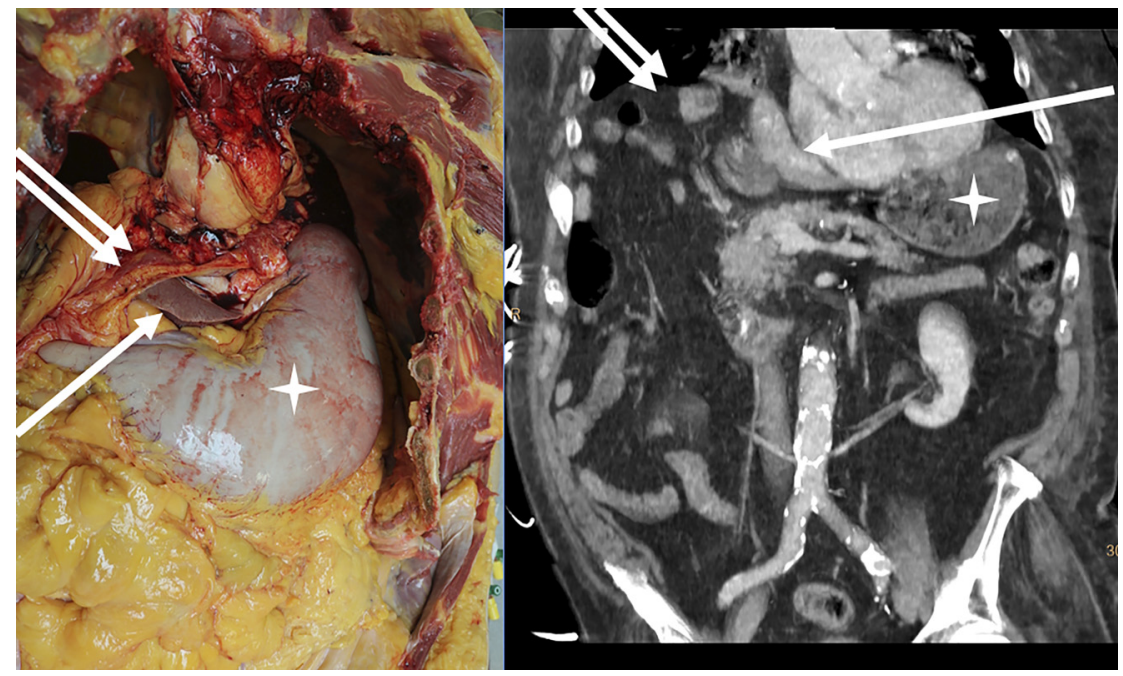

Fig. 4. Coronal plane CT reconstruction and autopsy images revealing the situation of the displaced and rotated liver (single arrow), and elevated diaphragm (double arrow), just behind the peritoneum and the gas filled stomach (asterisk). Left liver lobes are identical on both pictures

Funding sources: No financial support was received for this case report.
Conflict of interest: The authors have no conflict of interest to disclose. 


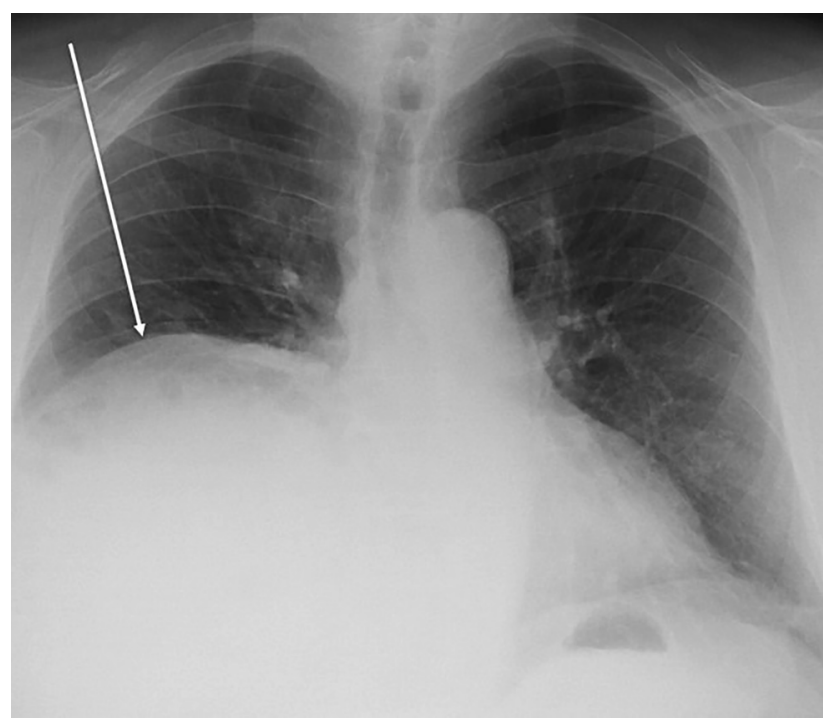

Fig. 5. Chest X-ray from 2014 (5 years before death) showing the right diaphragm already in a very high position leveling the pulmonary hilum. No gastric dilation is seen, a developmental malposition of the liver is also a possible cause of this unusual anatomy

\section{REFERENCES}

[1] Pepper PV, Goldstein MK: Postoperative complications in Parkinson's disease. J Am Geriatr Soc 1999; 47(8): 967-72.
[2] Leibson CL, Maraganore DM, Bower JH, Ransom JE, O'Brien PC, Rocca WA: Comorbid conditions associated with Parkinson's disease: a population-based study. Mov Disord 2006; 21(4): 446-55.

[3] Robbins J, Gensler G, Hind J, Logemann JA, Lindblad AS, Brandt $D$, et al.: Comparison of 2 interventions for liquid aspiration on pneumonia incidence: a randomized trial. Ann Intern Med 2008; 148(7): 509-18.

[4] Troche MS, Brandimore AE, Okun MS, Davenport PW, Hegland KW: Decreased cough sensitivity and aspiration pneumonia in Parkinson disease. Chest 2014; 146(5): 1294-9.

[5] Nobrega AC, Rodrigues B, Melo A: Is silent aspiration a risk factor for respiratory infection in Parkinson's disease patients? Parkinsonism Relat Disord 2008; 14(8): 646-8.

[6] Tanaka Y, Kato T, Nishida H, Araki H, Murase M, Nagaki M, et al.: Is there a difference in gastric emptying between Parkinson's disease patients under long-term L-dopa therapy with and without motor fluctuations? An analysis using the $13 \mathrm{C}$-acetate breath test. J Neurol 2009; 256(12): 1972-6.

[7] Tanaka Y, Kato T, Nishida H, Yamada M, Koumura A, Sakurai T, et al.: Is there a delayed gastric emptying of patients with earlystage, untreated Parkinson's disease? An analysis using the 13Cacetate breath test. J Neurol 2011; 258(3): 421-6.

[8] Heetun ZS, Quigley EM: Gastroparesis and Parkinson's disease: a systematic review. Parkinsonism Relat Disord 2012; 18(5): 433-40.

[9] Peker Y, Tatar F, Kahya MC, Cin N, Derici N, Reyhan E: Dislocation of three segments of the liver due to hernia of the right diaphragm. Hernia 2007; 11: 63-5.

[10] Miller T, Norman WT: Intermittent dislocation of the liver. Arch Surg 1977; 112: 658-62. 